\title{
NIH announces new strategy for research
}

\author{
Susan Mayor London
}

The US National Institutes of Health (NIH) announced a major strategy last week for enabling government funded medical research to meet modern challenges by making greater use of molecular biology and developing research teams that cross traditional boundaries.

Announcing what has become known as the NIH Roadmap, the director of the NIH, Elias Zirhouni, said: "As the 21st century unfolds, discovery in the life sciences is accelerating at an unprecedented rate. As science grows more complex, it is also converging on a set of unifying principles that link apparently disparate diseases through common biological pathways and therapeutic approaches. NIH research needs to reflect this new reality."

The roadmap has been devel- oped through discussions between the NIH, the scientific community, and the public over the past year. Participants were asked to look at three key issues: what are today's most pressing scientific challenges, what are the road blocks to progress, and what must be done to overcome these.

Working groups then translated the three main themes that emerged from these discussions-new pathways to discovery, research teams of the future, and re-engineering the clinical research enterprise-into 28 practical initiatives, resulting in the roadmap.

Initiatives in the section on new pathways to discoverydesigned to improve understanding of complex biological systems-aim to help researchers gain better access to information and techniques in genetics and molecular biology. They include measures to improve molecular imaging and the development of small molecular libraries.

The NIH will award grants for research in structural biology, development of metabolomics technology, and proteomics. In addition, it will support the development of new screening centres for bioactive small molecules, a publicly accessible chemi-informatics reference database (to be housed at NIH's National Center for Biotechnology Information), and a database and core facility dedicated to synthesising and distributing molecular imaging probes.

The agency will also begin planning a series of nanomedicine centres-carrying out quantitative measurement and manipulation of biological processes at the nanoscale or molecular level-which will be launched in 2005.

Measures designed to develop research teams of the future aim both to bring researchers from different disciplines together to solve complex biomedical research problems and to explore new organisational models for teams of scientists to work together.

Dr Zirhouni suggested that solving the puzzle of complex diseases would require understanding of the interplay of a range of factors. "We will need the expertise of non-traditional teams of biological scientists, engineers, mathematicians, physical scientists, computer scientists, and others."

Information on the NIH roadmap can be found at http:// nihroadmap.nih.gov

\section{Small improvement seen in teenager with vCJD}

Susan Mayor London

Neurologists have expressed cautious optimism about small improvements and lack of toxicity in a UK teenager with variant Creutzfeldt-Jakob disease (vCJD) treated with an experimental drug, pentosan polysulphate.

The patient, 19 year old Jonathan Simms from Belfast, Northern Ireland, has been treated with pentosan polysulphate by injection into the brain since a decision by the High Court last year that the potential benefits outweighed the risks (BMJ 2003;326:8).

Neurologists meeting in Belfast last week to review the early results of treatment considered that the drug seemed to be well tolerated and that it might have improved his condition.
After eight months' treatment, the patient had shown some improvement in neurological functions, including regaining the ability to swallow. His parents claimed that he had become more alert, responding to verbal instructions and attempting to speak. Monitoring of his heart rhythm showed less variation-thought to be associated with brain stem damage-than before starting treatment.

Nikolai Rainov, of the Walton Centre for Neurology and Neurosurgery, Liverpool, said: "The question of efficacy is still very much open. However, we believe that the brain stem now functions better than it did six to eight months ago." He reported: "Some neurological functions

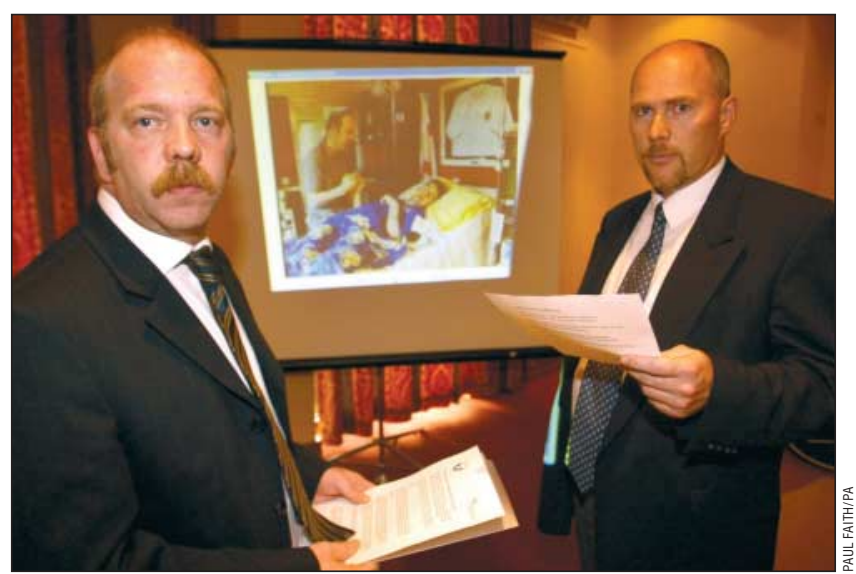

Don Simms (left) father of vCJD patient Jonathan Simms with the patient's GP, Dr Mark McClean, at the Stormont Hotel, Belfast

have improved significantly over the course of treatment. The patient is even able to obey commands-something which was not the case six months ago."

He considered that pentosan polysulphate might have a role in prolonging survival in patients with vCJD and in improving their quality of life. He added: "I believe that instigating treatment at an early stage could achieve significant extension of the life span of these patients." See News Extra at bmj.com 